British Journal of Nutrition (2022), 127, 148-149

(C) The Author(s), 2021. Published by Cambridge University Press on behalf of The Nutrition Society

\title{
Corrigendum
}

\section{Long-term effect of parental selenium supplementation on the one-carbon metabolism in rainbow trout (Oncorhynchus mykiss) fry exposed to hypoxic stress - CORRIGENDUM}

Pauline Wischhusen, Cécile Heraud, Kaja Skjærven, Sadasivam J. Kaushik, Benoit Fauconneau, Philip Antony Jesu Prabhu and Stéphanie Fontagné-Dicharry

DOI: https://doi.org/10.1017/S000711452100074X (First published online 04 March 2021)

The authors apologise for an error in the published paper, where by Tables 7 and 8 are identical. Table 7 in the published paper is correct.

The correct version of Table 8 is as follows:

Table 8. Essential and non-essential amino acids [ $\mu \mathrm{g} / \mathrm{g}$ sample] in muscle of fry originating from parents subjected to different Se treatments (Bnc, Bss, Bso) and then fed diets containing different levels and sources of Se (Fnc, Fss, Fso) measured in fish subjected to hypoxic stress.

\begin{tabular}{|c|c|c|c|c|c|c|c|c|c|}
\hline & \multicolumn{3}{|c|}{ Parental effect (PE) } & \multicolumn{3}{|c|}{ Direct feeding effect (FE) } & \multicolumn{3}{|c|}{$\mathrm{p}$-values } \\
\hline & $\mathrm{Bnc}$ & Bss & Bso & Fnc & Fss & Fso & PE & FE & PExFE \\
\hline $\operatorname{Arg}$ & $17 \pm 1$ & $18 \pm 1$ & $20 \pm 1$ & $19 \pm 1$ & $18 \pm 1$ & $18 \pm 1$ & 0.22 & 0.61 & 0.47 \\
\hline His & $552 \pm 12$ & $544 \pm 21$ & $560 \pm 14$ & $578 \pm 16$ & $544 \pm 23$ & $534 \pm 11$ & 0.81 & 0.21 & 0.45 \\
\hline Iso & $12 \pm 1$ & $13 \pm 1$ & $12 \pm 1$ & $14 \pm 1$ & $12 \pm 0$ & $12 \pm 1$ & 0.71 & 0.06 & 0.54 \\
\hline Leu & $31 \pm 2$ & $34 \pm 2$ & $31 \pm 2$ & $36 \pm 1^{\mathrm{a}}$ & $31 \pm 1^{\mathrm{ab}}$ & $29 \pm 2^{b}$ & 0.34 & 0.02 & 0.19 \\
\hline Lys & $13 \pm 3$ & $12 \pm 3$ & $14 \pm 3$ & $12 \pm 4$ & $11 \pm 2$ & $16 \pm 3$ & 0.79 & 0.46 & 0.14 \\
\hline Met & $13 \pm 1$ & $12 \pm 1$ & $12 \pm 1$ & $13 \pm 1$ & $12 \pm 1$ & $12 \pm 0$ & 0.36 & 0.15 & 0.49 \\
\hline Phe & $12 \pm 1$ & $11 \pm 0$ & $11 \pm 1$ & $12 \pm 1$ & $11 \pm 0$ & $11 \pm 0$ & 0.64 & 0.24 & 0.52 \\
\hline Thr & $71 \pm 4$ & $66 \pm 4$ & $71 \pm 4$ & $79 \pm 4^{\mathrm{a}}$ & $62 \pm 3^{b}$ & $67 \pm 3^{b}$ & 0.43 & $<0.01$ & 0.74 \\
\hline Val & $19 \pm 1$ & $20 \pm 1$ & $19 \pm 1$ & $22 \pm 1^{\mathrm{a}}$ & $18 \pm 1^{b}$ & $19 \pm 1^{a b}$ & 0.66 & 0.02 & 0.42 \\
\hline Ala & $262 \pm 4$ & $250 \pm 8$ & $261 \pm 9$ & $264 \pm 7$ & $252 \pm 7$ & $258 \pm 8$ & 0.46 & 0.54 & 0.47 \\
\hline Asn & $17 \pm 8$ & $38 \pm 12$ & $27 \pm 7$ & $36 \pm 9$ & $33 \pm 11$ & $14 \pm 6$ & 0.25 & 0.18 & 0.48 \\
\hline Asp & $43 \pm 4^{b}$ & $60 \pm 6^{a}$ & $45 \pm 3^{b}$ & $57 \pm 5^{\mathrm{a}}$ & $50 \pm 4^{\mathrm{ab}}$ & $40 \pm 4^{b}$ & 0.01 & 0.01 & 0.31 \\
\hline bAla & $71 \pm 4^{\mathrm{a}}$ & $52 \pm 3^{b}$ & $60 \pm 6^{a b}$ & $58 \pm 5$ & $60 \pm 5$ & $65 \pm 5$ & 0.03 & 0.52 & 0.70 \\
\hline Cit & $6 \pm 1$ & $6 \pm 0$ & $8 \pm 1$ & $7 \pm 1$ & $6 \pm 1$ & $6 \pm 1$ & 0.05 & 0.97 & 0.86 \\
\hline Gln & $70 \pm 7$ & $80 \pm 9$ & $77 \pm 3$ & $84 \pm 6$ & $78 \pm 6$ & $65 \pm 6$ & 0.42 & 0.08 & 0.16 \\
\hline Glu & $121 \pm 4$ & $120 \pm 9$ & $109 \pm 4$ & $117 \pm 7$ & $112 \pm 5$ & $120 \pm 6$ & 0.35 & 0.68 & 0.88 \\
\hline Gly & $404 \pm 21$ & $383 \pm 16$ & $442 \pm 19$ & $413 \pm 17$ & $400 \pm 26$ & $417 \pm 16$ & 0.08 & 0.77 & 0.13 \\
\hline Нур & $55 \pm 3$ & $51 \pm 4$ & $52 \pm 2$ & $55 \pm 3$ & $53 \pm 3$ & $50 \pm 3$ & 0.64 & 0.59 & 0.22 \\
\hline Orn & $4 \pm 0$ & $3 \pm 0$ & $4 \pm 0$ & $4 \pm 0^{\mathrm{a}}$ & $4 \pm 0^{\mathrm{ab}}$ & $3 \pm 0^{\mathrm{b}}$ & 0.30 & 0.05 & 0.02 \\
\hline Pro & $154 \pm 25$ & $139 \pm 14$ & $131 \pm 16$ & $181 \pm 17^{\mathrm{a}}$ & $137 \pm 14^{b}$ & $106 \pm 17^{b}$ & 0.43 & $<0.01$ & $<0.01$ \\
\hline Ser & $126 \pm 10$ & $138 \pm 10$ & $117 \pm 7$ & $138 \pm 11$ & $124 \pm 8$ & $119 \pm 9$ & 0.28 & 0.34 & 0.43 \\
\hline Tau & $1519 \pm 69$ & $1369 \pm 88$ & $1518 \pm 38$ & $1490 \pm 56$ & $1402 \pm 81$ & $1514 \pm 60$ & 0.17 & 0.42 & 0.27 \\
\hline Tyr & $11 \pm 1$ & $11 \pm 0$ & $11 \pm 1$ & $12 \pm 1$ & $11 \pm 0$ & $11 \pm 1$ & 0.39 & 0.06 & 0.39 \\
\hline AABA & $3 \pm 0$ & $3 \pm 0$ & $3 \pm 0$ & $4 \pm 0$ & $3 \pm 0$ & $3 \pm 0$ & 0.82 & 0.07 & 0.77 \\
\hline $\mathrm{NH} 4 \mathrm{Cl}$ & $104 \pm 2$ & $102 \pm 5$ & $101 \pm 2$ & $106 \pm 2$ & $100 \pm 4$ & $101 \pm 3$ & 0.84 & 0.42 & 0.33 \\
\hline BAA & $7 \pm 0^{\mathrm{ab}}$ & $6 \pm 0^{b}$ & $7 \pm 0^{a}$ & $6 \pm 0$ & $6 \pm 0$ & $7 \pm 0$ & 0.02 & 0.52 & 0.65 \\
\hline Ans & $1126 \pm 26$ & $1072 \pm 57$ & $1228 \pm 30$ & $1151 \pm 38$ & $1086 \pm 54$ & $1190 \pm 31$ & 0.40 & 0.47 & 0.83 \\
\hline Car & $75 \pm 11$ & $60 \pm 8$ & $66 \pm 9$ & $74 \pm 10$ & $73 \pm 6$ & $54 \pm 10$ & 0.41 & 0.15 & 0.07 \\
\hline Cysta & $9 \pm 2$ & $7 \pm 1$ & $9 \pm 2$ & $10 \pm 2$ & $8 \pm 1$ & $6 \pm 2$ & 0.57 & 0.31 & 0.28 \\
\hline $1 \mathrm{MHis}$ & $17 \pm 2$ & $16 \pm 2$ & $13 \pm 2$ & $13 \pm 2$ & $14 \pm 2$ & $19 \pm 3$ & 0.40 & 0.15 & 0.36 \\
\hline
\end{tabular}


Table 8. (Continued)

\begin{tabular}{|c|c|c|c|c|c|c|c|c|c|}
\hline & \multicolumn{3}{|c|}{ Parental effect (PE) } & \multicolumn{3}{|c|}{ Direct feeding effect (FE) } & \multicolumn{3}{|c|}{$p$-values } \\
\hline & Bnc & Bss & Bso & Fnc & Fss & Fso & PE & FE & PExFE \\
\hline PEA & $15 \pm 1$ & $14 \pm 1$ & $15 \pm 0$ & $15 \pm 1$ & $14 \pm 1$ & $15 \pm 0$ & 0.13 & 0.12 & 0.63 \\
\hline Sar & $9 \pm 1^{a b}$ & $12 \pm 1^{a}$ & $8 \pm 1^{b}$ & $10 \pm 1$ & $8 \pm 1$ & $11 \pm 1$ & $<0.01$ & 0.12 & 0.38 \\
\hline Urea & $1140 \pm 66$ & $1196 \pm 115$ & $920 \pm 92$ & $1038 \pm 139$ & $1154 \pm 61$ & $1064 \pm 74$ & 0.11 & 0.65 & 0.40 \\
\hline
\end{tabular}

Values are means \pm SEM $(n=9)$. Within columns and groups values not sharing a common superscript letter are significantly different according to two-way ANOVA followed by TukeyHSD.

Essential amino acids (EAA); Non-essential amino acids (NEAA); Arginine (Arg); Histidine (His); Isoleucine (Iso); Leucine (Leu); Lysine (Lys); Phenylalanine (Phe); Threonine (Thr); Valine (Val); Alanine (Ala); Aspartic acid (Asp); bAla (b-Alanine); Cit (Citrulline); Glutamine (Gln); Glutamic acid (Glu);Glycine (Gly); Hydroxy-L-Proline (Hyp); Ornithine (Orn); Proline (Pro); Serine (Ser); Taurin (Tau); Tyrosine (Tyr); L-alfa-amino-n-butyric acid (AABA); DL-beta-aminoisobutyric acid (BAA); Anserine (Ans); Carnosine (Car); Cystathionine (Cysta); 1-Methyl-L-Histidine (1MHis); O-Phosphoethanolamine (PEA); Sarcosine (Sar) 